\title{
Response of the Hypothalamic-Pituitary- Adrenal Axis to Stress in the Absence and Presence of Ethanol in Subjects at High and Low Risk of Alcoholism
}

Xing Dai, MD, M.Sc., Joseph Thavundayil, M.D., and Christina Gianoulakis, Ph.D.

Both genetic and environmental factors, such as stress, are important in determining alcohol consumption.

Furthermore, both stress and alcohol influence the activity of the hypothalamic-pituitary-adrenal (HPA)-axis. Thus, the present studies investigated the response of the HPA axis to stress and the effect of ethanol on the stress response, in subjects at high (HR) and low (LR) risk of alcoholism as determined from their family history. Twenty HR and 20 LR subjects performed a stress-inducing task $30 \mathrm{~min}$ following the ingestion of either a placebo drink or a low dose of ethanol. The levels of plasma adrenal corticotropic hormone (ACTH) and cortisol were measured prior to and for four hours following initiation of the treatment. Changes with time in the plasma hormone levels following ingestion of either a placebo or an ethanol drink, without the performance of the stress task, served as controls to compare the stress-induced changes. Neither the placebo nor the ethanol drink altered the plasma ACTH and cortisol concentrations. High risk subjects presented lower basal ACTH, but not cortisol, levels and a lower stress-induced increase in plasma ACTH concentration than LR subjects. Furthermore, the HR subjects presented a delayed poststress recovery of the plasma ACTH and cortisol levels. Ethanol consumption prior to the stress task attenuated (ACTH) or abolished (cortisol) the stress-induced increase in the plasma hormone concentrations of both $L R$ and $H R$ subjects. Thus, there are quantitative differences on the response of the HPA-axis to stress between $H R$ and $L R$ subjects, while ingestion of low amounts of ethanol prior to the performance of the stress task had a similar effect on $H R$ and LR individuals.

[Neuropsychopharmacology 27:442-452, 2002]

(C) 2002 American College of Neuropsychopharmacology.

Published by Elsevier Science Inc.
From the Douglas Hospital Research Centre and the Department of Psychiatry, McGill University, Montreal, Quebec, Canada.

The studies in the present manuscript were presented at the Annual Meeting of the Society for Neuroscience, on November 6, 2000, at New Orleans LA. Abstracts Society for Neuroscience 2000: Vol. 26, Abstract \# 300.10, p.803.

Address correspondence to: Christina Gianoulakis, Ph.D., Douglas Hospital Research Centre, 6875 LaSalle Blvd, Verdun, Quebec, Canada H4H 1R3. Tel.: (514) 761-6131, ext 5929; Fax:(514) 762-3034; E-mail: christina.gianoulakis@mcgill.ca

Received July 24, 2001; revised November 26, 2001; accepted February 8, 2002.

Online publication: 2/14/02 at www.acnp.org/citations/Npp 021402245 .
KEY WORDS: Ethanol; Stress; ACTH; Cortisol; HPA-axis; Family history of alcoholism

Experimental evidence indicates that both genetic and environmental factors play a significant role in determining alcohol consumption (Light et al. 1996). Studies using human subjects and experimental animals have demonstrated that stress is one of the environmental factors associated with the initiation and continuation of heavy drinking, as well as relapse (Hore 1971; Kushner et al. 1990; Pohorecky 1991; De Wit 1996; Le et al. 1998; Stewart 2000). Indeed, it has been shown that alcohol consumption increases in response to stress if 
other forms of support, such as social and family support, are not present (Pohorecky 1991; Johnson and Jennison 1994). The mechanisms underlying the relationship between stress and alcohol consumption are not well understood. It has been proposed that alcohol consumption relieves anxiety and thus may help the individual to cope with stress (Hodgson et al. 1979; Vogel and De Turck 1983; Pohorecky 1991). However, a number of studies indicate that alcohol can either reduce or produce anxiety (Cappell and Herman 1972; Hodgson et al. 1979; Malford 1983; Cappell and Greely 1987; Pohorecky 1991), depending on the experimental conditions used, such as the dose of alcohol (Klatsky et al. 1977; De Turck and Vogel 1982; Kelbaek et al. 1985), prior exposure to alcohol (Ireland et al. 1984; Marmot 1984) and whether or not the subject was stressed at the time of the study (Pohorecky 1981, 1991).

In designing studies investigating how ethanol influences stress responses, it is reasonable to investigate biological systems affected by both ethanol and stress. One such system is the hypothalamic-pituitary-adrenal (HPA)-axis the activity of which is enhanced by both ethanol and stress. In human, the major hormones of the HPA-axis are the hypothalamic corticotropin releasing hormone $(\mathrm{CRH})$, the pituitary adrenal corticotropic hormone (ACTH) and cortisol from the adrenal cortex. $\mathrm{CRH}$ stimulates cells of the anterior pituitary to synthesize and release ACTH, which in turn stimulates the synthesis and release of cortisol by the adrenal cortex. Furthermore, both CRH and cortisol have been reported to influence drinking behavior (Fahlke et al. 1995; Koob and Britton 1996; Erb et al. 1998; Le et al. 1998). Accepting that components of the HPA-axis can modulate alcohol consumption, then genetic or environmental factors that influence the activity of the HPA-axis may be important in determining a person's drinking behavior. Therefore, it may be proposed that inherited differences in the response of the HPA-axis to stress may predispose an individual to either heavier or lighter alcohol consumption.

Animal studies have consistently shown that the activity of the HPA-axis increases following alcohol consumption (Pohorecky 1981). However, human studies have indicated that ingestion of high, but not low, amounts of alcohol elevate plasma cortisol levels (Schuckit et al. 1987; Gianoulakis et al. 1996). Furthermore, sons of alcoholics presented a lower increase in plasma ACTH content in response to an alcohol challenge than sons of non-alcoholics (Schuckit et al. 1988) indicating inherited differences in the response of the HPA-axis to alcohol. Interestingly, it is the low doses of alcohol that reduce anxiety, while the high doses of alcohol not only do not reduce but may induce anxiety (Cappell and Herman 1972; Hodgson et al. 1979; De Turck and Vogel 1982; Malford 1983; Cappell and Greely 1987; Lukas and Mendelson 1988). Thus, it would be important to investigate whether ingestion of low amounts of alcohol, which do not significantly alter the activity of the HPA-axis, would influence the HPAaxis response to stress.

Thus, the objective of the present studies was to investigate the following hypotheses: (1) that there are differences in the response of the HPA-axis to stress between subjects at low (LR) and high (HR) risk for the future development of alcoholism as determined from their family history; (2) that ingestion of low amounts of alcohol, which when taken alone do not significantly alter the activity of the HPA-axis, will influence the HPA-axis response to stress of both HR and LR subjects; and (3) that the effect of alcohol on the stress response will be different between HR and LR subjects.

\section{Subjects and Methods}

HR and LR subjects were recruited from the Montreal area through advertisements placed in local newspapers and bulletin boards at local colleges and universities. The investigations were approved by the Research Ethics Board of the Douglas Hospital for human studies and were conducted in accordance with the guidelines of the Declaration of Helsinki. Informed consent was obtained from all subjects and they could withdraw from the study at any time.

\section{Subject Selection}

All subjects were males between 19 and 25 years old and were not alcoholics at the time of testing. All subjects were screened for the presence of family history of alcoholism by a structured interview of as many family members as possible, administered by a trained research assistant, and the construction of a family pedigree. For the subjects as well as for other family members agreeing to be interviewed, the diagnosis of alcoholism was based on a score greater than 10 on the MAST (Selzer 1971) and the presence of alcohol dependence as determined by the computerized diagnostic interview schedule (DIS) of the DSM-IV (American Psychiatric Association 1994). For family members who were not available for interview (those who were dead, living outside Montreal or were unwilling to be interviewed) the Family History Research Criteria were used (Endicot et al. 1975). Subjects whose father and paternal grandfather were alcoholics (two generations history of alcoholism) made up the HR group, while subjects with no alcoholic members in their family for the two last generations made up the LR group. Although the presence of alcoholism in the family of the mother was not a necessary criterion it was not excluded. However, subjects whose mother was, or had previously been, an alcoholic were excluded from the study to avoid including subjects exposed to ethanol 
during fetal life. Fetal ethanol exposure has been shown to alter the response of the offspring to stress (Angelogianni and Gianoulakis 1989; Weinberg et al. 1986; Taylor et al. 1986).

All subjects were given a complete medical examination, including blood biochemistry, hematology and urine analysis. The psychopathology and personality traits of the subjects, such as antisocial personality disorder, were assessed using the Diagnostic Interview Schedule of the DSM-IV (American Psychiatric Association 1994). Furthermore, to investigate the quantity, frequency and variability of drinking behavior, a detailed drinking history of the last three months was obtained from each subject (Cahalan and Cisin 1968). Exclusion criteria were: (1) a MAST score of 10 or higher (Selzer 1971) and the presence of alcohol dependence or abuse diagnosis (DSM-IV) (American Psychiatric Association 1994); (2) medical problems, such as liver, kidney, diabetes, hypertension or other chronic disease; (3) mental health problems such as depression and schizophrenia; (4) use of prescribed psychotropic drugs; (5) use of other drugs of abuse such as cocaine and cannabis; and (6) smoking of more than 10 cigarettes per day. Subjects were matched for age, education, smoking, years of drinking, daily and weekly alcohol consumption.

Since genetic vulnerability to alcoholism is also transmitted to daughters from either their fathers or mothers (Kendler et al. 1994), both daughters and sons of alcoholic fathers could have inherited the vulnerability to alcoholism and could be included in the HR group. However, female subjects were not included in the present studies due to the complexity of controlling for the phases of the reproductive cycle and the use of various contraceptive medications. Instead, another study has been designed controlling for those factors in female HR and LR subjects.

\section{Outline of the Experimental Procedures}

The objective of the proposed research was to investigate whether there are genetically determined differences among HR and LR individuals on the response of the HPA-axis following exposure to stress in the absence and presence of ethanol. Each subject participated in four testing sessions in a random order. The minimum interval between testing sessions was seven days. All subjects were requested to abstain from alcohol for $48 \mathrm{~h}$ prior to each testing session as determined by selfreport and estimation of the blood alcohol content.

Session 1: Subjects received a placebo drink to determine the presence of differences in the basal activity of the HPA-axis as a function of family history.

Session 2: Subjects ingested a low quantity of ethanol $(0.50 \mathrm{~g}$ ethanol $/ \mathrm{kg}$ body weight) to determine its effect on the activity of the HPA-axis as a function of family history.

Session 3: Subjects received a placebo drink and 30 min later they performed a stress-inducing task (Matthews et al. 1987) to determine the presence of differences on the response of the HPA-axis to stress as a function of family history.

Session 4: Subjects were given an ethanol drink $(0.50 \mathrm{~g}$ ethanol $/ \mathrm{kg}$ body weight) $30 \mathrm{~min}$ prior to the performance of the stress-inducing task, to determine (1) whether alcohol prior to stress would modulate the stress response of the HPA-axis and (2) whether this ethanol effect on the stress response would be different between HR and LR subjects.

On the day of testing subjects arrived at the clinical research unit of Douglas Hospital between 7:30 and 8:00 A.M. An intravenous catheter was placed in the nondominant arm vein and the subjects were offered a light breakfast consisting of two slices of toast without butter or jam, to avoid the feeling of nausea. Nausea could act as a stressor, increasing the release of $\mathrm{CRH}, \mathrm{ACTH}$ and cortisol, making it difficult to distinguish the effect of the experimental procedures on these hormones. Subjects could drink water with their breakfast but not coffee or tea. Also subjects could smoke prior to their arrival at the clinical research unit, but not during the experimental session. At 45 and $60 \mathrm{~min}$ following positioning of the catheter (15 and $0 \mathrm{~min}$ prior to the ingestion of the placebo or the alcohol drink depending on the experimental session) blood samples were drawn to estimate the basal ACTH and cortisol levels. Then subjects received the appropriate treatment, depending on the testing session. Additional blood samples were obtained every $15 \mathrm{~min}$ for the first hour and every $30 \mathrm{~min}$ for the second, third and fourth hour post-treatment. A total of $12 \times 5 \mathrm{ml}$ blood samples were obtained from each subject in each testing session for estimation of the plasma ACTH and cortisol concentrations.

Based on power calculations from data obtained in preliminary experiments, the study was designed to include $20 \mathrm{HR}$ and $20 \mathrm{LR}$ subjects. In the course of the study one HR and one LR subject dropped out for personal reasons. Their matched pairs were also withdrawn and new matched pairs entered the study. Thus, $22 \mathrm{HR}$ and $22 \mathrm{LR}$ subjects initiated the study but only 20 subjects from each risk group completed the four experimental sessions.

\section{Description of the Stress Task}

The subjects were required to solve mentally a number of mathematical problems, under the pressure of time and competition, for monetary bonuses to be awarded to the five highest scorers out of every 10 subjects completing the study. The duration of the stress task was 10 
min. This test was chosen because it has been shown to act as a psychological stressor (Matthews et al. 1987).

\section{Preparation of the Placebo and Alcohol Drinks}

Studies have indicated that it is the ingestion of low doses of ethanol, which do not significantly alter the plasma cortisol or ACTH levels, that reduce anxiety and induce a general feeling of well being (Cappell and Herman 1972; Hodgson et al. 1979; De Turck and Vogel 1982; Malford 1983; Cappell and Greely 1987; Lukas and Mendelson 1988). Thus, it was the objective of the present studies to investigate the effect of a low dose of ethanol on the activity of the HPA-axis and its response to stress. The dose of $0.5 \mathrm{~g}$ per $\mathrm{kg}$ body weight was chosen since previous studies have shown that it does not induce significant changes in the plasma cortisol and ACTH concentrations (Schuckit et al. 1987, 1988; Lukas and Mendelson 1988; Gianoulakis et al. 1996). The alcohol drink consisted of 1 part 95\% USP ethanol and 2 parts unsweetened orange juice. The placebo drink consisted of 1 part degassed tonic water and 2 parts unsweetened orange juice. The lips of the glass were dipped in alcohol to give it the smell and taste of alcohol. The beverage was consumed as a single drink within $5 \mathrm{~min}$. Subjects initially could not distinguish between the placebo and alcohol drinks. However, in the absence of the pharmacological effects of ethanol some subjects did suspect that they had consumed a placebo drink.

\section{Estimation of the Blood Alcohol Content}

To estimate the blood alcohol content (BAC) a commercial kit based on the alcohol dehydrogenase method (Hawkins et al. 1966) was used following the instructions of the supplier (SIGMA NAD-ADH Kit No 332-B. Sigma Chemical Company, St. Louis. Mo).

\section{Estimation of Plasma ACTH and Cortisol Contents}

The blood samples were collected in heparinized vacutainer tubes containing $1 \mathrm{mM}$ N-ethylmaleimide and were centrifuged immediately at 3,000 $\times \mathrm{g}$ for $15 \mathrm{~min}$ in a refrigerated centrifuge. The plasma was stored at $-75^{\circ} \mathrm{C}$ for estimation of the plasma ACTH and cortisol contents. The plasma ACTH content was estimated using a commercial radioimmunoassay Kit (catalog number 40-2195, Nichols Institute Diagnostics, San Juan Capistrano, CA). Results are reported as pg ACTH $/ \mathrm{ml}$ plasma. The sensitivity of this ACTH assay is $1 \mathrm{pg}$ $\mathrm{ACTH} / \mathrm{ml}$. The intra-assay and interassay coefficients of variation were $3.2 \%$ and $7.8 \%$ respectively.

The content of total plasma cortisol was estimated using the AMERLEX Cortisol radioimmunoassay kit (catalog number 8758401; Ortho-Clinical Diagnostics,
Inc. Rochester, NY). Results are reported as $\mu \mathrm{g}$ cortisol/ $100 \mathrm{ml}$ plasma. The sensitivity of this cortisol assay is $0.1 \mu \mathrm{g} / 100 \mathrm{ml}$. The intra-assay and interassay coefficients of variation were $4.3 \%$ and $7.7 \%$ respectively.

\section{STATISTICAL ANALYSIS}

For statistical evaluation a 3-way mixed repeated measures analysis of variance (ANOVA) with Risk as the independent factor and with Time and Experimental session as the correlated factors was used. When significant main or interactive effects were indicated by the 3-way ANOVA, post-hoc analysis was performed using the Newman-Keuls multiple comparison test, known to control for the inflated $\alpha$ level which results from multiple comparisons. In some occasions the Fisher's LSD, a less conservative test that does not control for the inflated $\alpha$ level, was used. The significance was evaluated at a level of 0.05 . In addition, where appropriate, data was analyzed by the 2-tailed student's $t$-test or 1-way and 2-way ANOVAs. All statistical analyses were performed using the DATASIM statistical software package (DATASIM Version 1.1, 1996) for an IBM compatible computer.

\section{RESULTS}

\section{Subject Description}

The major characteristics of the $20 \mathrm{HR}$ and 20 LR subjects who completed the study are shown in Table 1. The HR and LR subjects were matched for age, body weight, education, smoking and drinking behavior such as age at onset, quantity and frequency of drinking. None of the subjects were drinking excessively or were alcoholics at the time of testing.

Table 1. Subject Description

\begin{tabular}{lcc}
\hline Parameter & Low Risk & High Risk \\
\hline Number of subjects & 20 & 20 \\
Age & $22.05 \pm 2.60^{*}$ & $22.5 \pm 2.80$ \\
Body weight $(\mathrm{kg})$ & $76.75 \pm 11.70$ & $81.35 \pm 16.80$ \\
Years of education & $14.3 \pm 2.60$ & $12.7 \pm 2.40$ \\
Alcoholic relatives & 0.00 & $3.7 \pm 1.50$ \\
Drinking days per month & $3.35 \pm 2.30$ & $3.55 \pm 2.76$ \\
Drinks per drinking day & $2.85 \pm 1.70$ & $4.10 \pm 2.3$ \\
Age at onset of drinking & $16.15 \pm 1.53$ & $15.55 \pm 3.05$ \\
Antisocial personality disorder & & \\
$\quad$ ASPD) & 0.00 & 0.00 \\
Michigan alcoholism screening & & \\
$\quad$ test (MAST)** & $2.00 \pm 1.20$ & $1.90 \pm 1.90$ \\
\% Smokers & 30 & 30 \\
Cigarettes per day & $5.33 \pm 0.80$ & $5.55 \pm 0.81$ \\
\hline
\end{tabular}

${ }^{*}$ Mean \pm SEM

**A MAST score higher than 5 indicates a problem drinker. 


\section{Blood Alcohol Content}

The changes with time of the BAC of HR and LR subjects following ingestion of $0.5 \mathrm{~g}$ ethanol $/ \mathrm{kg}$ body weight. were similar to those observed in our previous studies (Gianoulakis et al. 1996). Maximum BAC, in $\mathrm{mmol} / \mathrm{L}$ blood, was obtained at $30 \mathrm{~min}$ post-ethanol, $14.0 \pm 0.7$ for LR and $12.8 \pm 1.1$ for the HR subjects, and then the BAC presented a gradual decrease to $4.3 \pm 0.3$ for LR and $4.1 \pm 0.6$ for HR at the end of the experimental session ( $4 \mathrm{~h}$ post-ethanol). Statistical evaluation of the data using a 3-way ANOVA (Risk $\times$ Time $\times$ Experimental session) indicated a significant main effect of Time but no significant main effects of either Risk or Experimental session.

\section{Basal ACTH and Cortisol Levels}

Each HR and LR subject participated in four experimental sessions at different days given in a random order. From each subject we received blood samples at 15 and $0 \mathrm{~min}$ prior to the ingestion of the placebo or alcohol drink to estimate the plasma hormone levels under basal conditions. At the initiation of blood sampling some subjects may present stress-induced elevations in the plasma hormone concentrations due to unfamiliarity with the clinical procedure. Thus, to investigate the presence of differences in the basal plasma ACTH and cortisol levels between HR and LR subjects the hormone concentrations detected at $0 \mathrm{~min}$ of each of the four experimental sessions were used. Figure 1 presents both a bar graph and a scatter graph of the plasma concentrations of ACTH and cortisol under basal conditions. The HR subjects presented lower basal ACTH levels $(p<.00012$-tailed student's $t$-test) than the LR subjects. However, despite the lower basal ACTH levels observed in HR compared with LR subjects, the plasma cortisol levels were similar between the two risk groups. Furthermore, for each subject the basal levels of each hormone were grouped in the order of visits. Statistical evaluation using 1-way ANOVA indicated no significant effect of visit order for either LR or HR subjects. This lack of effect may be due to two factors: (1) the subjects were familiar with the research unit from previous visits (medical exam and lab test or participation in other studies by the alcohol research program) and (2) the catheter was inserted one hour prior to drawing of the basal blood sample.

\section{Effects of the Four Treatments on Plasma ACTH Levels}

Figure 2, panels $\mathrm{A}$ and $\mathrm{B}$, demonstrates the changes in plasma ACTH concentration under the four experimental conditions in LR and HR subjects respectively. The 3-way ANOVA indicated a significant main effect of

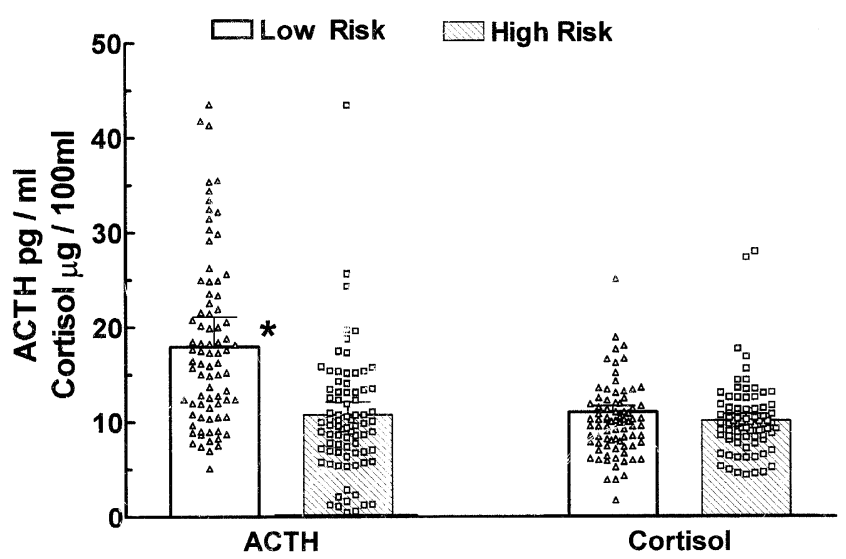

Figure 1. Scatter and bar graphs of the basal levels of plasma ACTH and cortisol in low risk (open bars) and high risk (striped bars) subjects. Bars represent the mean \pm SEM of $20 \mathrm{LR}$ and $20 \mathrm{HR}$ subjects. Each scatter point represents the hormone concentration at $0 \mathrm{~min}$ for each experimental session and subject (four scatter points for each subject, one for each experimental session). *Significant difference between low and high risk subjects $p<.0001$ (2-tailed students $t$-test).

Risk factor $\left(\mathrm{F}_{1,1919}=22.19 ; p<.0001\right)$. There was no significant main effect of either Time or Treatment factors; however, there was a significant interaction between the Treatment and Time factors $\left(\mathrm{F}_{33,1919}=2.46 ; p<.0001\right)$.

Figure 2, panels $\mathrm{A}$ and $\mathrm{B}$, demonstrates that there was no significant change in the plasma ACTH concentration following ingestion of either the placebo or the alcohol drink by both LR and HR subjects, in agreement with the absence of a significant main effect of time in the 3-way ANOVA. Furthermore, Figure 2 demonstrates that HR subjects have lower plasma ACTH levels than LR subjects, in agreement with the significant main effect of Risk factor in the 3-way ANOVA. Posthoc analysis using the Newman-Keul's multiple comparison test, at a 0.05 level of significance, indicated significant differences in the ACTH levels between LR and HR subjects at all time points tested of the placebo and alcohol sessions and at most time points of the placebostress ( 0 to $150 \mathrm{~min}$ ) and alcohol-stress (0 to $120 \mathrm{~min}$ ) sessions $(p<.05$ Newman-Keuls).

Figure 2 demonstrates an increase in the plasma ACTH concentration as early as $15 \mathrm{~min}$ post-stress by both LR and HR subjects, in agreement with the significant interaction between Time and Treatment factors in the 3-way ANOVA. When the stress task was performed 30 min following ingestion of the placebo drink, in the LR subjects the maximum stress-induced increase in plasma ACTH concentration was observed at $45 \mathrm{~min}$ (15 min post stress) and was significantly higher from the pre-stress concentrations at 0 and $30 \mathrm{~min}(p<.05$ Newman-Keuls). Subsequently, the plasma ACTH concentration presented a gradual decrease, and at about 


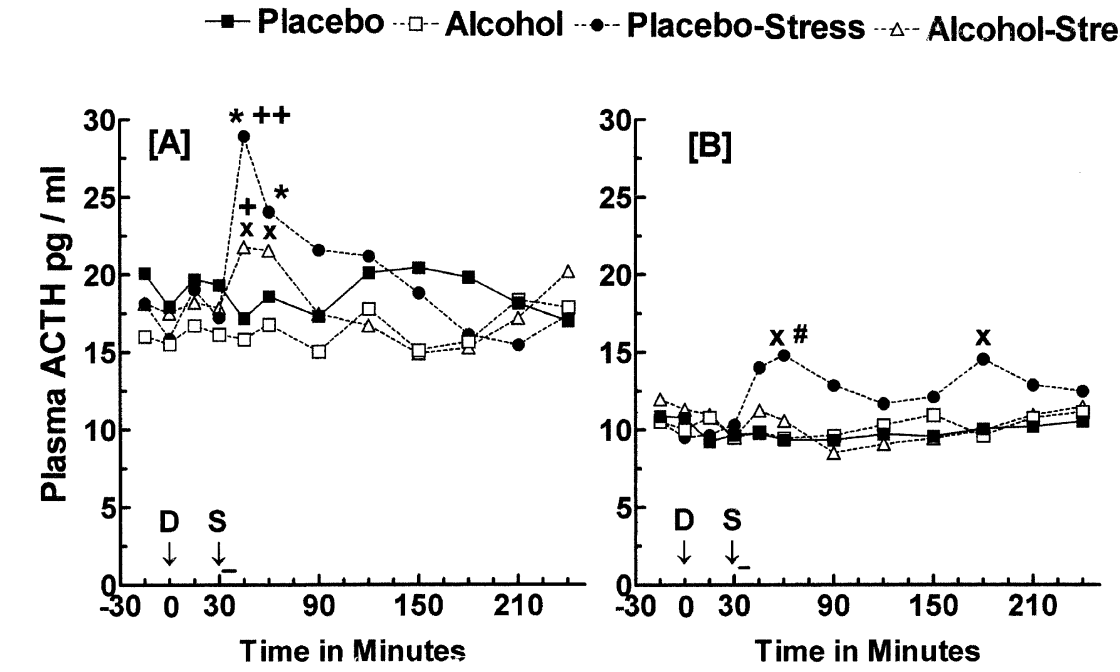

-- Placebo-Stress $-\Delta-$ - Alcohol-Stress

Figure 2. Changes in the plasma ACTH concentration with time under the four experimental conditions. Arrows indicate (D) the time of ingestion of the placebo or alcohol drink and (S) the time of initiation of the stress task, which lasted for 10 min. Each point represents the mean of 20 subjects. For better clarity of the graph the standard errors of the means are not shown. *Significantly different from the pre-stress levels at 0 and $30 \min p<.05$; Newman-Keuls multiple comparison test. $\mathbf{x}$ Significantly different from the pre-stress levels at 0 and $30 \mathrm{~min} p<.05$ Fisher's LSD multiple comparison test. ${ }^{+}$Significantly different from the placebo-only session (its corresponding control treatment) at the same time points $p<.05$; Newman-Keuls multiple comparison test. ${ }^{+}$Significantly different from the alcohol-only session (its corresponding control treatment) at the same points $p<.05$; Fisher's LSD multiple comparison test. \#Significantly different from the placebo-only session (its corresponding control treatment) at the same points $p<.05$ Fisher's LSD multiple comparison test.

150 min it was similar to the pre-stress levels. In the HR subjects the maximum stress-induced increase in plasma ACTH concentration was observed at $60 \mathrm{~min}$ (30 min post-stress) and was significantly different from the pre-stress levels at 0 and $30 \mathrm{~min}(p<.05$ Fisher's LSD). Subsequently, there was a gradual decrease toward the pre-stress levels. However, contrary to the case with the low risk subjects the ACTH levels remained higher than the pre-stress levels and at 180 min the ACTH concentration was significantly higher than that at 0 min $(p<.05$ Fisher's LSD).

When the stress task was performed 30 min following the ingestion of the alcohol drink in the LR subjects the plasma ACTH concentration was higher from the pre-stress levels at 45 and $60 \mathrm{~min}$ (15 and $30 \mathrm{~min}$ poststress) $(p<.05$ Fisher LSD), but was lower than that observed when the stress task was performed $30 \mathrm{~min}$ following ingestion of the placebo drink ( $p<.05$ Newman-Keuls). In the HR group a small elevation in the plasma ACTH concentration was observed, which, however, was not significantly different from the prestress levels. These observations indicate that ingestion of the alcohol drink prior to stress significantly attenuated the stress-induced increase in plasma ACTH concentration of both LR and HR subjects.

Since the 3-way ANOVA indicated a significant interaction between Treatment and Time factors, it was important to test whether the stress-induced changes in plasma ACTH concentration were significantly different from the concentrations at the same time points of the corresponding control session, which for the placebo-stress treatment was the placebo-only session and for the alcohol-stress treatment was the alcohol-only session. Thus, for each risk group post-hoc analysis comparing the ACTH concentrations at distinct time points among the four experimental sessions was performed. Results indicated that in the LR group the plasma ACTH concentration at $45 \mathrm{~min}$ (15 min poststress) of the placebo-stress session was significantly higher from that of its corresponding control treatment, the placebo-only session ( $p<.05$ Newman-Keuls). Furthermore, at $45 \mathrm{~min}$ of the alcohol-stress session the ACTH concentration was significantly different from its corresponding control treatment, the alcohol-only session $(p<.05$ Fisher's LSD). Similar post-hoc analysis in the HR group indicated that at $60 \mathrm{~min}(30 \mathrm{~min}$ poststress) the ACTH concentration of the placebo-stress session was significantly different from that of its corresponding control treatment, the placebo-only session ( $p$ $<$.05 Fisher's LSD). However, at $60 \mathrm{~min}$ of the alcoholstress session, the ACTH concentration was not significantly different from its corresponding control treatment, the alcohol-only session. These observations suggest that consumption of the alcohol drink prior to stress attenuated the stress-induced increase in plasma ACTH concentration for both LR and HR subjects. 
The difference in the ACTH concentration between HR and LR subjects during the placebo-stress and alcohol-stress sessions are a combination of the differences in the pre-stress ACTH concentrations and the differences in the ACTH response to stress. To distinguish whether the ACTH response to stress by the LR group was significantly different from that of the HR group, the maximum net stress-induced increase was estimated by subtracting the pre-stress ACTH concentration from the maximum post-stress concentration. Thus, in the LR subjects the maximum net increase at 15 min post-stress was $11.70 \pm 2.99$ in the placebo stress session and $3.92 \pm 1.88$ in the alcohol-stress session. In the HR subjects the maximum net increase at $30 \mathrm{~min}$ post-stress was $4.46 \pm 1.72$ in the placebo-stress session and $1.08 \pm 0.88$ in the alcohol-stress session. A 2-way ANOVA (Risk $X$ Treatment) was performed and indicated a significant main effect of Risk $\left(\mathrm{F}_{1,76}=7.691 p<\right.$ $.007)$ and Treatment $\left(\mathrm{F}_{1,76}=6.268 ; p<.0144\right)$. Post-hoc analysis using the Newman-Keuls multiple comparison test indicated that following the placebo drink the maximum post-stress increase in plasma ACTH concentration of the LR subjects was significantly higher from that of the HR subjects, $p<.05$. However, following the alcohol drink the stress-induced increase of plasma ACTH concentration in LR subjects, was not significantly different from that observed in HR subjects.

\section{Effect of the Four Treatments on Plasma Cortisol Levels}

Figure 3, panels A and B, demonstrates the changes in plasma cortisol levels under the four experimental conditions in LR and HR subjects. The 3-way ANOVA indicated a significant main effect of Time $\left(\mathrm{F}_{11,1919}=18.49 ; p<\right.$ .0001) and a significant interaction between Treatment and Time factors $\left(\mathrm{F}_{33,1919}=3.61 ; p<.0001\right)$. There was no significant main effect of Risk factor in agreement with the similar plasma cortisol concentrations in the two risk groups of subjects, however there was a significant interaction between Risk and Time factors $\left(\mathrm{F}_{11,1919}=\right.$ $2.38 ; p<.0064)$.

Figure 3 demonstrates that following the placebo drink there was a gradual decrease in the plasma cortisol levels of both LR and HR subjects in agreement with the significant main effect of Time. Post-hoc analysis to compare differences in the plasma cortisol concentration between distinct time points of the placebo session indicated that the plasma cortisol concentration at 0 min was significantly higher from those at 60, 90, 120, 180,210 and $240 \mathrm{~min}(p<.05$ Newman-Keuls) in the LR group and from those at 60, 90 and 240 in the HR group $(p<.05$ Newman-Keuls). Following ingestion of the alcohol drink a small non-significant increase was observed at $15 \mathrm{~min}$ in the LR but not HR group which was followed by a gradual decrease so that at $240 \mathrm{~min}$ the plasma cortisol concentration of both LR and HR subjects was significantly lower from that at $0 \min (p<.05$ Fisher's LSD).

As shown on Figure 3, panels A and B, performance of the stress task $30 \mathrm{~min}$ following ingestion of the placebo drink induced a fast increase in the plasma cortisol levels reaching a maximum at $60 \mathrm{~min}$ (30 min poststress) in both LR and HR subjects in agreement with the significant interaction between Time and Treatment factors obtained in the 3-way ANOVA. In the LR group post-hoc analysis indicated that the cortisol concentration at 45 and $60 \mathrm{~min}$ (15 and $30 \mathrm{~min}$ post-stress) was significantly higher from the pre-stress levels at 0 and $30 \min (p<.05$ Newman-Keuls). Subsequently, there was a gradual decrease so that at $150 \mathrm{~min}$ the plasma cortisol concentration had returned to pre-stress levels and at $240 \mathrm{~min}$ was significantly lower from the concentration at $0 \mathrm{~min}(p<.05$ Fisher's LSD). In the HR group post-hoc analysis indicated that the plasma cortisol concentration at $60 \mathrm{~min}$ was significantly higher from the pre-stress levels at $30 \mathrm{~min}(p<.05$ Newman-Keuls). Subsequently, there was a gradual decrease so that between 120-240 min the plasma cortisol concentrations were similar to the pre-stress levels.

As is clearly shown on Figure 3, panels A and B, when the stress task was performed $30 \mathrm{~min}$ following ingestion of the alcohol drink there was no significant stress-induced increase in plasma cortisol concentration by either LR or HR subjects. Instead, as was the case when the alcohol drink was administered alone, there was a gradual decrease in plasma cortisol levels so that at 180 and $210 \mathrm{~min}$ the plasma cortisol concentrations were significantly lower from the basal levels at $0 \mathrm{~min}$ $(p<.05$ Newman-Keuls) for both HR and LR subjects.

Since the 3-way ANOVA indicated a significant interaction between Treatment and Time factors, it is important to test whether the stress-induced changes in plasma cortisol concentration were significantly different from the concentrations at the same time points of the corresponding control session, which for the placebo-stress treatment was the placebo-only session and for the alcohol-stress treatment was the alcohol-only session. Thus, further post-hoc analysis was performed comparing the cortisol concentrations at distinct time points among the four experimental sessions. Results indicated that the cortisol concentration of the placebo stress-session at 60 min (30 min post-stress) was significantly higher from the concentration of its corresponding control treatment, the placebo-only session, in both LR $(p<.05$ NewmanKeuls) and HR ( $p<.05$ Fisher's LSD). On the other hand, for both LR and HR subjects, the cortisol concentration of the alcohol-stress session at $60 \mathrm{~min}$ was not significantly different from the concentration of its corresponding control treatment, the alcohol-only session.

The 3-way ANOVA did not demonstrate a significant main effect of Risk factor; however, it did indicate 

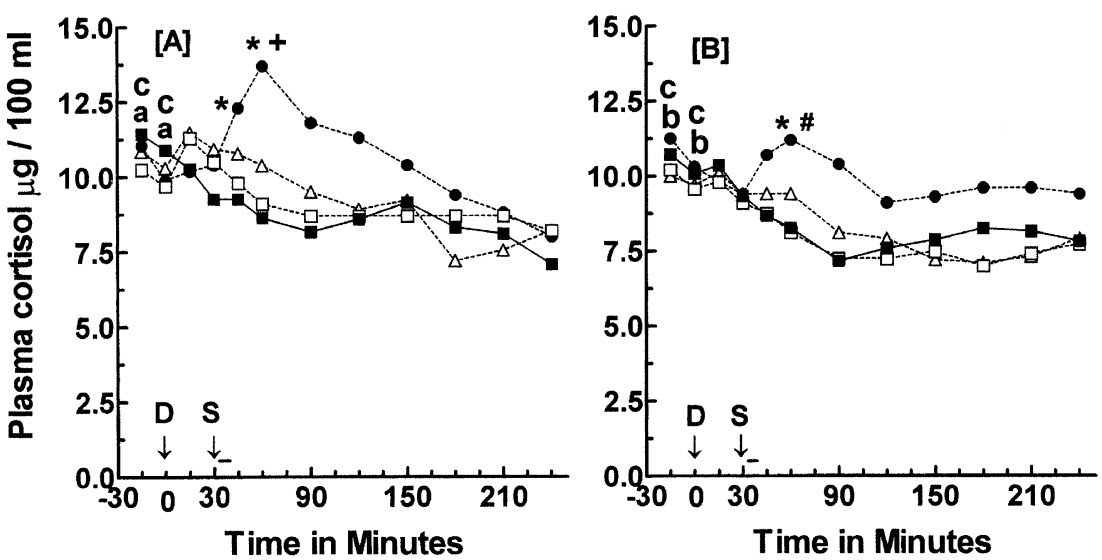

Figure 3. Changes in the plasma cortisol concentration with time under the four experimental conditions. Arrows indicate (D) the time of ingestion of the placebo or alcohol drink and (S) the time of initiation of the stress task, which lasted for 10 min. Each point represents the mean of 20 subjects. For better clarity of the graph the standard errors of the means are not shown. a Significantly different from the cortisol concentration at 60, 90, 120, 180, 210, and 240 min of the same session (placebo) $p<.05$ Newman-Keuls multiple comparison test. b Significantly different from the 60, 90, and 240 min of the same session (placebo) $p<.05$ Newman-Keuls multiple comparison test. c Significantly different from 240 min of the same experimental session (alcohol) $p<.05$ Fisher's LSD multiple comparison test. *Significantly different from the pre-stress levels $(30 \mathrm{~min}) p<.05$ Newman-Keuls multiple comparison test. ${ }^{+}$Significantly different from the placebo-only session (its corresponding control treatment) at the same time points $p<.05$ Newman-Keuls multiple comparison test. \#Significantly different from the placebo-only session (its corresponding control treatment) $p<.05$ Fisher's LSD multiple comparison test.

a significant interaction of Risk with Treatment factors. Thus post-hoc analysis was performed to investigate the presence of significant simple effects of the Risk factor on the Treatment and Time factors at a 0.05 level of significance. Results indicated that in the placebo-stress session at $60 \mathrm{~min}$ (30 min post-stress) the cortisol concentration of the LR subjects was significantly higher than that of the HR subjects ( $p<.05$ Fisher's LSD). This observation suggests that in the LR subjects, stress (in the absence of prior alcohol ingestion) induces a more pronounced increase in plasma cortisol levels than in the HR subjects.

\section{DISCUSSION}

Among the major findings was the presence of differences in the response of the HPA-axis to stress as a function of family history of alcoholism. Thus, the LR subjects presented a higher increase of plasma ACTH and cortisol in response to stress than the HR subjects. In addition, ingestion of low amounts of ethanol prior to the performance of the stress task attenuated the HPA-axis response to stress in both LR and HR subjects.

Possible mechanisms responsible for the lower ACTH release in response to stress by the HR than LR subjects could be: (1) a lower responsivity of the ACTH producing cells to $\mathrm{CRH}$; and (2) a lower release of CRH in response to stress. Previous studies have shown a lower sensitivity of the pituitary producing cells to exogenous (ovine) CRH in the sons of alcoholic (Waltman et al. 1994) than non-alcoholic parents. This observation supports the hypothesis of a lower sensitivity of the ACTH producing cells to endogenous $\mathrm{CRH}$ in the HR compared with the LR subjects. However, a lower stress-induced CRH release, as well as the presence of a central CRH deficiency in the HR subjects cannot be excluded. Indeed, a lower ACTH response to stress associated with central CRH deficiency has been observed in the alcohol consuming Lewis rats compared with the Fisher rats, which do not voluntarily drink alcohol (Beitner-Johnson et al. 1991).

The earlier return to the basal ACTH levels, or even below the basal levels, observed in the LR compared with HR subjects, may be due, at least in part, to the higher stress-induced increases in the plasma ACTH and cortisol concentrations in the LR subjects. Higher increases in plasma ACTH and cortisol levels could exert a stronger negative feedback and thus, a faster inhibition of central CRH release. This faster decrease in central CRH release would lead to a faster decrease in the ACTH release in the LR than HR subjects and as result to a faster return to the pre-stress or even below the 
pre-stress levels. Therefore, in HR subjects the longer lasting post-stress elevations of ACTH levels may be attributed to the lower stress-induced elevations in plasma ACTH and cortisol levels, inducing a weaker negative feedback on central CRH release. Studies have shown that central CRH levels may play a significant role in increasing alcohol consumption by both human and experimental animals (Koob and Britton 1996; Le et al. 1998; Stewart 2000). Thus, the stress-induced elevations of central CRH levels may be responsible, at least in part, for the increased alcohol consumption associated with stressful life events.

An important observation in the present studies was that the basal plasma ACTH levels were significantly lower in the HR than LR subjects, while the basal plasma cortisol levels were similar between the HR and LR subjects. These observations are in agreement with previous studies demonstrating lower basal plasma $\beta$-endorphin (Gianoulakis et al. 1996) and ACTH (Waltman et al. 1994) concentrations in HR than LR subjects, but similar plasma cortisol concentrations (Waltman et al. 1994; Gianoulakis et al. 1996). The mechanisms accounting for this dissociation between ACTH and cortisol concentrations are not clear. The lower basal ACTH levels in HR than LR subjects may be due, at least in part, to a more sensitive negative feedback by cortisol at the level of the brain and pituitary gland in HR than LR subjects. Alternatively, they may also be accounted for by an earlier occurrence of the ACTH circadian peak in HR than LR subjects. However, the absence of significant differences in the circadian rhythms of ACTH and cortisol between HR and LR subjects have been reported (Wand et al. 1999).

The present data demonstrates that following ingestion of the placebo drink there was a gradual decrease in the plasma cortisol levels for the initial 90-120 min of the experimental session, but not of plasma ACTH levels, suggesting a lack of correlation between the changes of plasma ACTH and cortisol with time for both HR and LR subjects. This reduction in plasma cortisol levels with time may reflect either a normal circadian pattern or elevation of plasma cortisol levels prior to the initiation of blood sampling $(-15 \mathrm{~min})$ due to other factors such as insertion of the catheter and consumption of the light meal. However, since stress should have also increased the plasma ACTH levels, the decrease in plasma cortisol concentration with time is most likely due to the normal circadian pattern of the hormone. In addition, previous studies in our laboratory (unpublished data) demonstrated that a regular meal, but not a light snack, induced a transient increase in plasma ACTH and cortisol levels, which however had returned to basal values within $60 \mathrm{~min}$. The long resting period following the placement of the catheter and consumption of the light meal, as well as the familiarity of the subjects with the clinical research unit and its personnel, from their previous visits during the medical exam and laboratory tests, may account for the lack of significant stress-induced elevations in the plasma hormone levels at the beginning of the experimental session. The absence of changes in ACTH concentration with time may indicate that the circadian ACTH peak ends earlier than the circadian cortisol peak for both LR and HR subjects.

Heavy cigarette smoking is known to alter the activity of the HPA axis, therefore, it was one of the exclusion criteria in the present studies. Compared with nonsmokers, individuals smoking more than 25 cigarettes per day presented higher plasma cortisol and lower plasma ACTH levels at 9:00 A.M. (Del Arbol et al. 2000). However, the plasma ACTH and cortisol levels of individuals smoking 10 cigarettes or less per day were similar to those of non-smoking control subjects (Del Arbol et al. 2000). Furthermore, it has been reported that following the smoking of two cigarettes within $10 \mathrm{~min}$ the plasma ACTH and cortisol levels increased, reached maximum levels within $20 \mathrm{~min}$ and returned to basal values within 60 to $90 \mathrm{~min}$ (Coiro and Vescovi 1999). Thus, subjects smoking 10 or less cigarettes per day were included in the present studies. In addition, they were allowed to smoke prior to their arrival at the clinical research unit, but not during the experimental session. The time interval between the arrival of the subject at the clinical research unit and the initiation of the experimental session (consumption of the placebo or alcohol drink) was about $90 \mathrm{~min}$ (clinical admission of the subject, positioning of the catheter, consumption of the light breakfast and the $60 \mathrm{~min}$ rest period). Furthermore, the HR and LR subjects were matched for smoking including the same number of smokers in each group. In the present studies, comparison of the hormone levels between smokers and non-smokers within each risk group indicated the absence of significant differences.

Prior to testing the effect of an ethanol drink on the stress-induced activation of the HPA axis it was important to test the effect of the low dose of ethanol chosen to be used in the present studies on the activity of the HPA-axis in the absence of stress. It was speculated that this dose of ethanol would not be sufficient to increase the plasma levels of ACTH and cortisol, but would be sufficient to modulate the response of the HPA-axis to stress. Indeed, the results of the present studies indicated that the changes in plasma ACTH and cortisol concentrations following ingestion of the low dose of ethanol were similar to those observed following ingestion of the placebo drink in both HR and LR subjects. However, when the same quantity of ethanol was taken 30 min prior to the performance of the stress task, it did influence the response of the HPA axis to stress.

Thus, when the ethanol drink was consumed $30 \mathrm{~min}$ prior to the stress task, it attenuated the stress-induced 
increase in plasma ACTH levels and abolished the stress-induced increase in the plasma cortisol levels, of both HR and LR subjects. This reduction in the ACTH and cortisol responses to stress following alcohol ingestion could be due to a decreased responsivity of the ACTH producing cells to CRH in the presence of alcohol. Indeed, previous studies have reported that in human alcohol blunted the ACTH response to exogenous (ovine) CRH (Waltman et al. 1993). Alternatively, in the presence of ethanol stress may induce the release of lower amounts of CRH due either to an ethanol induced inhibition of factors which stimulate $\mathrm{CRH}$ release such as serotonin and norepinephrine, or to an ethanol induced stimulation of factors which inhibit CRH release, such as opioid peptides and $\gamma$-aminobutyric acid (GABA) (Gianoulakis 1990; De Waele et al. 1992; Wand et al. 1998). Further studies should be performed to elucidate the mechanisms and neurotransmitter systems involved in the ethanol induced attenuation of the HPA axis response to stress.

This property of ethanol to decrease the stressinduced activation of the HPA axis may play a significant role in promoting alcohol consumption. Thus, assuming that the extent and duration of the increases in plasma cortisol and ACTH contents indicate how stressful an individual perceives a specific situation, then attenuation or prevention of the stress-induced increases in the plasma hormone contents by specific interventions, such as an alcohol drink, may indicate that following the intervention the individual perceives the same situation as being less or non-stressful. This ability of ethanol to relieve stress and reduce anxiety may be one of the reasons that stressful experiences are usually associated with increased alcohol consumption.

Despite the possible relationship of the alcohol induced changes in the hormonal responses to stress with decreased anxiety, subjective measures of anxiety at distinct time points of the experimental session were not taken in the present studies. It was considered that completing a questionnaire could act as an additional mild stressor, at least for some individuals, and could alter the pattern of the post-stress recovery of the hormone levels. However, knowledge of the subjective feelings and mood changes of the subjects under the various experimental conditions could have allowed a more accurate interpretation of the data. Lack of such information is considered as a limitation of the present studies.

In summary, the present studies demonstrate that there are quantitative differences on the response of the HPA axis to stress between HR and LR subjects, while ingestion of low amounts of ethanol prior to the stress task attenuated the HPA axis response to stress in both HR and LR subjects. Thus, a small quantity of alcohol prior to stress may induce a similar anxiolytic effect in LR and HR subjects, and may explain why stress in- creases alcohol consumption by both HR and LR individuals. Future studies should investigate the effect of ethanol consumption after the performance of the stress task, as well as of higher doses of ethanol.

\section{ACKNOWLEDGMENTS}

This work was supported by the Canadian Institutes on Health Research. The authors wish to thank Ms Sandra Santella and the nursing staff of the Clinical Research Unit of Douglas Hospital for their expert assistance.

The studies in the present manuscript were presented at the Annual Meeting of the Society for Neuroscience, on November 6, 2000, at New Orleans, LA. Abstracts Society for Neuroscience 2000: Vol. 26, Abstract \# 300.10, p.803.

\section{REFERENCES}

American Psychiatric Association (1994): Diagnostic and Statistical Manual of Mental Disorders (DSM-IV). Washington, DC, American Psychiatric Association

Angelogianni P, Gianoulakis C (1989): Prenatal exposure to ethanol alters the ontogeny of the $\beta$-endorphin response to stress. Alcohol Clin Exp Res 13:564-571

Beitner-Johnson D, Guitart X, Nestler EJ (1991): Dopaminergic brain regions of Lewis and Fisher rats display different levels of tyrosine hydroxylase and other morphineand cocaine- regulated phosphoproteins. Brain Res 561:147-150

Cappell H, Greely J (1987): Alcohol and tension reduction: an update on research and theory. In Blane HT, Leonard HE (eds), Psychological Theories of Drinking and Alcoholism. Guilford Press, New York, NY, pp 15-24

Cappell H, Herman CP (1972): Alcohol and tension reduction: A review. Quart J Stud Alcohol 33:33-64

Cahalan D, Cisin H (1968): American drinking practices: Summary from a national probability sample. J Stud Alcohol 29:130-139

Coiro V, Vescovi PP (1999): Effect of cigarette smoking on $\mathrm{ACTH} /$ cortisol secretion in alcoholics after short- and medium-term abstinence. Alcohol Clin Exp Res 23:1515-1518

Del Arbol JL, Raya Munoz J, Ojeda L, Lopez Cascales A, Irles JR, Miranda MT, Ruiz Requena ME, Aguirre JC (2000): Plasma concentrations of beta-endorphin in smokers who consume different numbers of cigarettes per day. Pharmacol Biochem Behav 67:25-28

De Turck KH, Vogel WH (1982): Effects of acute ethanol on plasma and brain catecholamine levels in stressed and unstressed rats: evidence for ethanol-stress interaction. J Pharmacol Exp Ther 223:348-354

De Waele JP, Papachristou DN, Gianoulakis C (1992): The alcohol-preferring C57BL/6 mice present an enhanced sensitivity of the hypothalamic $\beta$-endorphin system to ethanol than the alcohol-avoiding DBA/2 mice. J Pharmacol Exp Ther 261:788-794

De Wit H (1996): Priming effects with drugs and other reinforcers. Exp Clin Psychopharmacol 4:5-10 
Endicot J, Andreasen N, Spitzer RL (1975): Family History Research Diagnostic Criteria. New York, NY, Biometric Research New York State Psychiatric Institute.

Erb S, Shaham Y, Stewart J (1998): The role of corticotropinreleasing factors and corticosterone in stress-cocaineinduced relapse to cocaine seeking in rats. J Neurosci 18:5529-5536

Fahlke C, Hard E, Eriksson CJP, Engel JA, Hansen S (1995): Consequence of long-term exposure to corticosterone or dexamethasone on ethanol consumption in adrenalectomized rat, and the effect of type I and II corticosteroid receptor antagonists. Psychopharmacology (Berl) 117: 216-224

Gianoulakis C (1990): Characterization of the effects of acute ethanol administration on the release of $\beta$-endorphin peptides by the rat hypothalamus. Eur J Pharmacol 180:21-29

Gianoulakis C, Krishnan B, Thavundayil J (1996): Enhanced sensitivity of pituitary beta- endorphin to ethanol in subjects at high risk of alcoholism. Arch Gen Psychiatry 53:250-257

Hawkins RD, Kalant H, Khana JM (1966): Effects of chronic intake of ethanol on the rate of ethanol metabolism. Can J Physiol Pharmacol. 44:241-257

Hodgson RJ, Stockwell TR, Rankin HJ (1979): Can alcohol reduce tension? Behav Res Ther 17:459-466

Hore BD (1971): Factors in alcohol relapse. Br J Addiction to Alcohol and Other Drugs. 66:89-96

Ireland MA, Vandongen R, Davidson L, Beilin LJ, Rouse IL (1984): Acute effects of moderate alcohol consumption on blood pressure and catecholamines. Clin Sci. 66:643-648

Johnson KA, Jennison KM (1994): Stressful loss and the buffering of social support on drinking behavior among African Americans: Results of a national survey. J Alcohol Drug Educ. 30:1-24

Kelbaek H, Gjorup T, Brynjolf I, Christensen NJ, Godtfredsen J (1985): Acute effects of alcohol on left ventricular function in healthy subjects at rest and during upright exercise. Am J Cardiol 55:164-167

Kendler KS, Neale MC, Heath AC, Kessler RC, Eaves LJ (1994): A twin-family study of alcoholism in women. Am J Psychiatry 151:707-715

Klatsky AL, Friedman GD, Siegelaub AB, Gerard MJ (1977): Alcohol consumption and blood pressure. Kaiser-Permanente Multiphasic Health Examination Data. N J Med 296:1194-1200

Koob FG, Britton KT (1996): Neurobiological substrates for anti-anxiety effects of ethanol. In Begleiter $\mathrm{H}$ and Kissin B (eds), The Pharmacology of Alcohol and Alcohol Dependence. New York, Oxford University Press, pp 477-506

Kushner MG, Sher KJ, Beitman BD (1990): The relation between alcohol problems and anxiety disorders. Am J Psychiatry 147:685-695

Le AD, Quan B, Juzytch W, Fletcher PJ, Joharchi N, Shaham Y (1998): Reinstatement of alcohol-seeking by priming injections of alcohol and exposure to stress in rats. Psychopharmacology (Berl) 135:169-174

Light JM, Irvine KM, Kjerulf L (1996): Estimating genetic and environmental effects of alcohol use and dependence from a national survey: a quasi-adoption study. J Stud Alcohol 57:507-520

Lukas SE, Mendelson JH (1988): Electroencephalographic activity and plasma ACTH during ethanol-induced euphoria. Biol Psychiatry 23:141-148

Marmot MG (1984): Alcohol and coronary heart disease (review). Intern J Epidemiol 13:160-167

Matthews KA, Rakaczky CJ, Stoney CM, Manuck SB (1987): Are cardiovascular responses to behavioral stressors a stable individual difference variable in childhood? Psychophysiology 24:464-473

Malford HA (1983): Stress, alcohol intake and problem drinking in Iowa. In Pohorecky LA, Bricks J (eds), Stress and Alcohol Use. Elsevier Biomedicals, New York, pp 321-335

Pohorecky LA (1981): The interaction of alcohol and stress (review). Neurosci Behav Rev 5:209-229

Pohorecky LA (1991): Stress and alcohol interaction: an update of human research. Alcohol Clin Exp Res 15:438-495

Selzer ML (1971): The Michigan Alcoholism Screening Test: The quest for a new diagnostic instrument. Am J Psychiatry 127:1653-1658

Schuckit MA, Gold EO, Risch C (1987): Plasma cortisol levels following ethanol in sons of alcoholics and controls. Arch Gen Psychiatry 44: 942-945

Schuckit MA, Risch SC, Gold EO (1988): Alcohol consumption, ACTH level and family history of alcoholism. Am J Psychiatry 145:1391-1395

Stewart J (2000): Pathways to relapse: The neurobiology of drug-and stress-induced relapse to drug-taking. J Psychiatry Neurosc 25:125-136

Taylor AN, Branch BJ, Nelson LR, Lane LA, Poland RE (1986): Prenatal ethanol and ontogeny of pituitary-adrenal responses to ethanol and morphine. Alcohol 3:255-259

Vogel WA, De Turck KH (1983): Stress and alcohol use. In Pohorecky LA, Bricks J (eds), Stress and Alcohol Use. New York, Elsevier Biomedical, pp 429-438

Waltman C, Blevis LS, Boyd G, Wand GS (1993): The effects of mild ethanol intoxication on the hypothalamic-pituitary-adrenal axis in non-alcoholic men. J Clin Endocrinol Metabol. 7:518-522

Waltman C, McCaul ME, Wand GS (1994): Adrenocorticotropin responses following administration of ethanol and ovine corticotropin-releasing hormone in the sons of alcoholics and control subjects. Alcohol Clin Exp Res 18:826-830

Wand GS, Mangold D, Ali M, Giggey P (1999): Adrenocortical responses and family history of alcoholism. Alcohol Clin Exp Res 23:1185-1190

Wand GS, Mangold D, El Diery S, McCaul ME, Hoover D (1998): Family history of alcoholism and hypothalamic opioidergic activity. Arch Gen Psychiatry 55:1114-1119

Weinberg J, Nelson LR, Taylor AN (1986): Hormonal effects of fetal alcohol exposure. In West JR (ed), Alcohol and Brain Development. New York, Oxford University Press, pp 310-342 